\title{
Concept for the Implementation of Very High Directivity and Decade Bandwidth in Compact Microstrip Directional Couplers
}

\author{
Alexander Alt ${ }^{* \dagger}$, Nikolai Schwerg ${ }^{\ddagger}$, Christian Wangler ${ }^{\dagger}$, Daniel Gruner ${ }^{\dagger}$ \\ * School of Engineering, Cardiff University, Cardiff, United Kingdom \\ $\dagger$ TRUMPF Huettinger GmbH \& Co. KG, Freiburg, Germany \\ $\ddagger$ CERN, Geneva, Switzerland
}

\begin{abstract}
A concept to induce a very high directivity and a flat coupling over a large bandwidth in compact microstrip directional couplers is presented. By introducing lumped element based compensation networks at the isolated and coupled port of a simple electrically short directional coupler, a very high directivity and a constant coupling over enormous bandwidths up to one decade can be achieved. With a design focusing on flat coupling, a high power directional coupler with a coupling length of only $50 \mathrm{~mm}$, less then $\lambda / 25$ at the middle of the band, was shown to have a coupling in the range of $52.11 \mathrm{~dB} \pm 0.05 \mathrm{~dB}$ from 37.4 MHz to $200 \mathrm{MHz}$. In a further design step with focus on directivity and coupling flatness at the same, the coupling stayed within $55.25 \mathrm{~dB} \pm 0.35 \mathrm{~dB}$ from $22 \mathrm{MHz}$ to $164 \mathrm{MHz}$ with a directivity of greater than $50 \mathrm{~dB}(30 \mathrm{~dB})$ from $35 \mathrm{MHz}$ to $84 \mathrm{MHz}$ (20 MHz to $187 \mathrm{MHz}$ ).
\end{abstract}

Index Terms-Directional coupler, microstrip circuits, power monitoring, reflection coefficient measurement

\section{INTRODUCTION}

Plasma processing tools for the fabrication of modern semiconductors require an extremely precise process control. For processes that are operating with radio-frequencies (RF) this results in the need for RF generators with an extremely high degree of accuracy over a wide dynamic range, e.g. a power repeatability for the same generator of $1 \%$ within a power range of $800-10,000 \mathrm{~W}$ as provided by TRUMPF Huettinger's new TruPlasma VHF series 3010 (G1/40) generator. The high power directional coupler, being one of the most important components within the power control loop of an $\mathrm{RF}$ generator, should meet various challenging requirements. A broadband performance is desired to address multiple operating frequencies and to integrate diagnostic features like the capturing of spurious emissions of the non-linear plasma load. Broadband performance means to achieve a constant coupling over a certain bandwidth. This enables the use of the full dynamic range of the subsequent RF detector circuit for all the targeted frequencies and translates into measurement resolution and accuracy. Furthermore, a constant coupling is important to protect the RF detector circuit, which could be damaged by more strongly coupled spectral emissions of the plasma chamber. A common way to realise a constant coupling for microstrip line couplers is the use of multi-sectional coupled lines. However, the coupled structure needs to have dimensions in the order of a quarter-wavelength and would

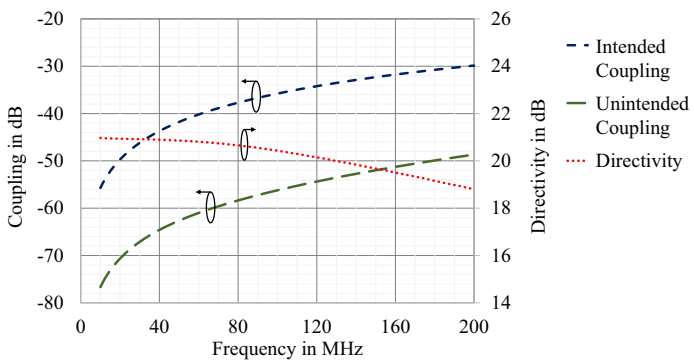

(a)

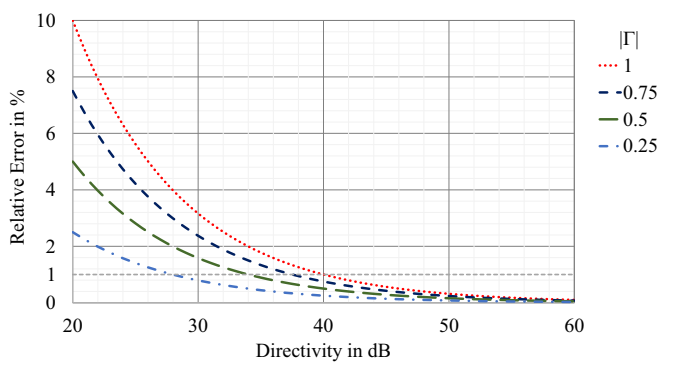

(b)

Fig. 1. (a) Coupling and Directivity of a typical microstrip directional coupler (b) Maximum measurement error due to directivity for different values of $|\Gamma|$

be far too large for operating frequencies in the HF and VHF range. Accordingly, electrically short couplers are commonly in use for the targeted applications and a coupling increasing with $6 \mathrm{~dB}$ per octave has to be addressed, cf. Fig. 1a. On the other hand, broadband coupler performance means to realise high directivity over a large frequency range. Apart from other effects like drift over power, temperature, life time etc. the directivity of the coupler is directly related to the measurement error as illustrated in Fig. 1b, showing the measurement error as a function of the directivity and the load reflection coefficient. Due to the dispersive nature of a microstrip coupler and the unequal phase velocities of even and odd mode, the directivity of a microstrip coupler is inherently limited. Several topologies to overcome this issue have been investigated like the introduction of a dielectric overlay, the use of wiggly [1],[2] or fragment-type [3] coupled lines or the use of a capacitive [4],[5] or inductive loading [6]. However, these methods partly are not sufficient or practically not useful 


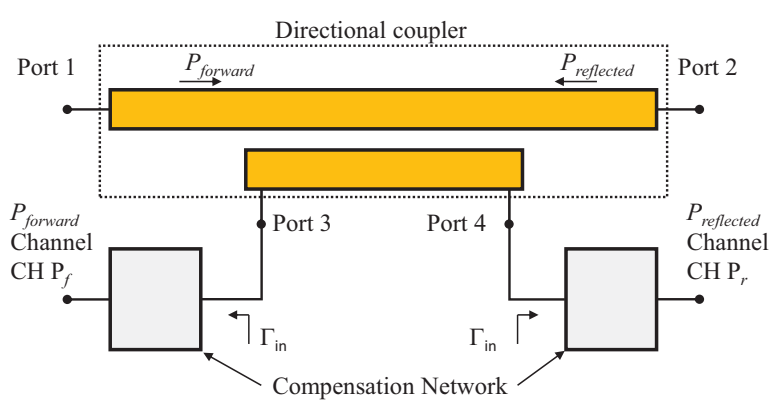

Fig. 2. Block diagram of the proposed concept

for the implementation of broadband, electrically-short high power directional couplers. Other topologies are focusing on voltage cancellation techniques like in [7], [8], [9] but do not address broadband performance or are rather complex by using two coupling lines. In contrast to that, the focus of this paper is the creation of a broadband electrically-short directional coupler with flat coupling and high directivity. The proposed concept uses lumped element compensation networks at the isolated and coupled port of a simple microstrip line coupler with only one coupled line. These networks are synthesised in a way to - 1) realise a voltage cancellation of the miscoupled power contribution over a large bandwidth resulting in a high directivity - 2) to compensate for the $6 \mathrm{~dB}$ per octave increase of the coupling at the same time. Both major design goals are first investigated independently from each other before bringing together the respective design considerations in a second step.

\section{CONCEPT}

The proposed concept requires a directional coupler with only one coupled line which is on both sides connected to the measurement system via a compensation network. Dependent on the requirements, this network can be designed to provide flat coupling, a high directivity or a trade-off between the two. The coupled line which has both sides terminated with a load network with an input impedance of $Z_{\text {in }}$ and an output impedance matched to the system impedance $Z_{\text {ref }}$, cf. Fig. 2 . This concept has two main challenges, high directivity and a flat coupling, that shall be treated separately. The high directivity is discussed first, coupling flatness is then treated followed by the combination of the two requirements.

\section{A. Inducing broadband directivity}

In [9] and [8], the principle of terminating one port of the coupled line with a carefully chosen impedance to improve the directivity is explored. The problem is that due to the limited directivity, the reflected power is partially coupled into the forward power port. By terminating the reflected port with a slightly mismatched impedance a small portion of power is reflected in the right phase to cancel out this miscoupled power at the forward port. This leads to a very high directivity but at a limited bandwidth, [8] reports $67 \mathrm{~dB}$ but the directivity stays only below $50 \mathrm{~dB}$ over a bandwidth of about $2 \%$. A similar

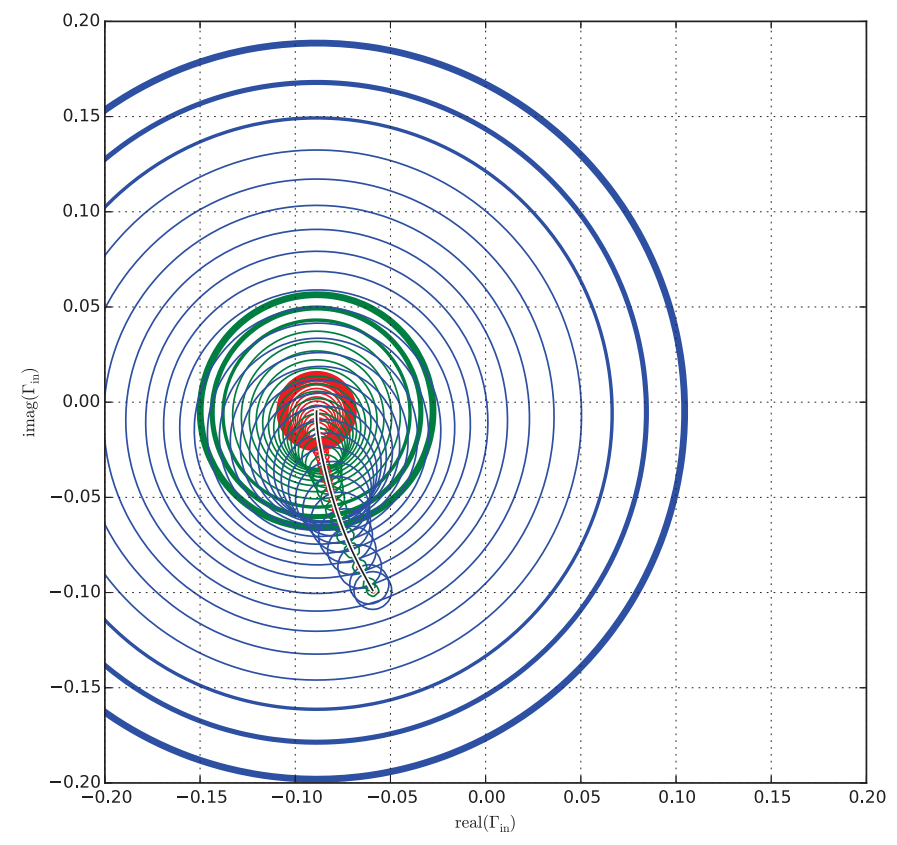

Fig. 3. Input reflection $\Gamma_{\text {in }}$ coefficients providing a given minimum isolation of the measurement port. The frequency increases from $10 \mathrm{MHz}$ to $200 \mathrm{MHz}$ from the larger to the smaller circles. The colours indicate increasing values of isolation from $80 \mathrm{~dB}$ (blue) to $100 \mathrm{~dB}$ (red). The solid black line shows the locus of the input reflection $\Gamma_{\text {in }}$ providing ideal isolation.

approach uses both forward and reflected power cancellation to achieve a very broadband directivity of above $40 \mathrm{~dB}$ [7]. The proposed approach shall follow the general idea of cancelling out by using reflections but extending it to work over a wider band.

We consider a directional coupler connected to two identical compensation networks as shown in Fig. 2. The directional coupler can be fully described by its frequency depending s-parameters which were determined either by simulation or measurements. The output of the compensation network shall be matched to the reference impedance of the measurements system $Z_{\text {ref. }}$ By means of choosing the input reflection coefficient $\Gamma_{\text {in }}$ we can maximise the isolation of the measurement ports. The choice of the $\Gamma_{\text {in }}$ defines, in turn, the electrical network of the compensation network.

With the given port numbering the intrinsic isolation of the directional coupler is given by $S_{32}$ describing the direct path between the opposite port 2 and the measurement port for the forward power, i.e. port 3 . With ports 3 and 4 closed we get two additional contributions $-\Gamma_{\text {in }} S_{32} \cdot S_{44}$ and $\Gamma_{\text {in }} \cdot S_{34} \cdot S_{42}$. Figure 3 shows the range of values of $\Gamma_{\text {in }}$ of the two networks in order to reach a certain isolation. For lower frequencies the circles are larger which is due to the fact that the intrinsic isolation of the directional coupler is higher for lower frequencies, cf. Fig. 1a and therefore the system is less sensitive to the compensation network. Setting the sum of all 3 contributions to zero, one can solve for the optimum reflection 


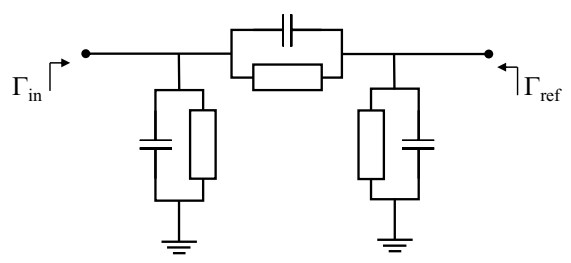

Fig. 4. Example for a directivity enhancement circuit

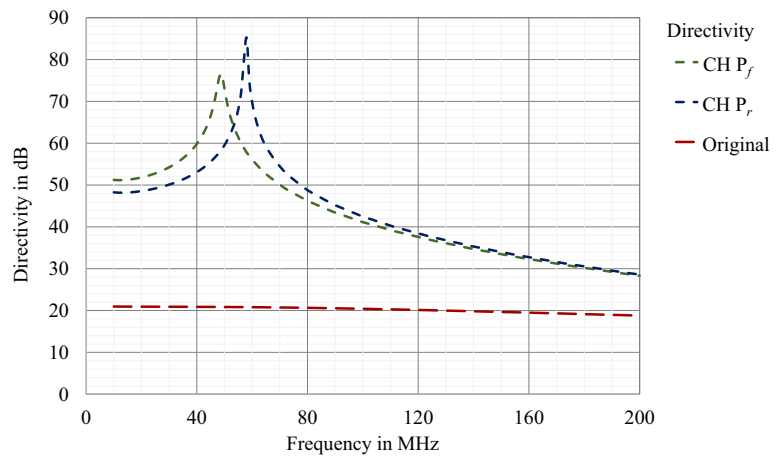

Fig. 5. Directivity with and without directivity enhancement circuit

coefficient as a function of frequency:

$$
\Gamma_{\mathrm{opt}}=\frac{S_{32}}{S_{32} \cdot S_{44}-S_{43} \cdot S_{42}}
$$

The locus of the optimum reflection coefficient resembles a counter-clockwise turning circle. This behaviour can be approximated partially by means of a compensation network as shown in Fig. 4 resulting in a directivity which remains above $40 \mathrm{~dB}$ for more than $100 \mathrm{MHz}$ and gives better than 50 dB for about $60 \mathrm{MHz}$, see Fig. 5 .

Due to small differences in the coupling and isolation values in the datasets of the directional coupler in Fig. 1a, the directivity of the two ports is different despite using the same compensation network. This could be rectified by adapting the compensation networks individually but the ability to use only one configuration for both ends is the key point to keep the concept simple as the networks influence each other.

\section{B. Equalising coupling over frequency}

To keep the circuit from introducing non-linearities, only passive components can be used. Therefore, the power above the level of lowest coupling $C_{L}$ needs to be reduced in order to bring the coupling to a constant level. As the impedances at both ports are to be equal, this cannot be achieved by reflecting the excess power, which thus leads to the introduction of a frequency dependent attenuator. As the coupling increases with frequency, cf. Fig. 1a, the circuit needs to attenuate more at higher frequencies. Starting with a $\Pi$ attenuator, the required behaviour can be achieved by including RC series impedance in parallel to the shunt resistors and a RL series impedance in parallel with the series resistor. The values depend on the intended bandwidth and thus the intended level of attenuation

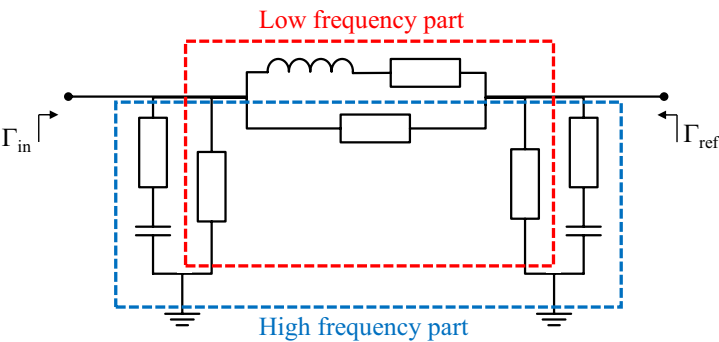

Fig. 6. Proposed coupling compensation circuit

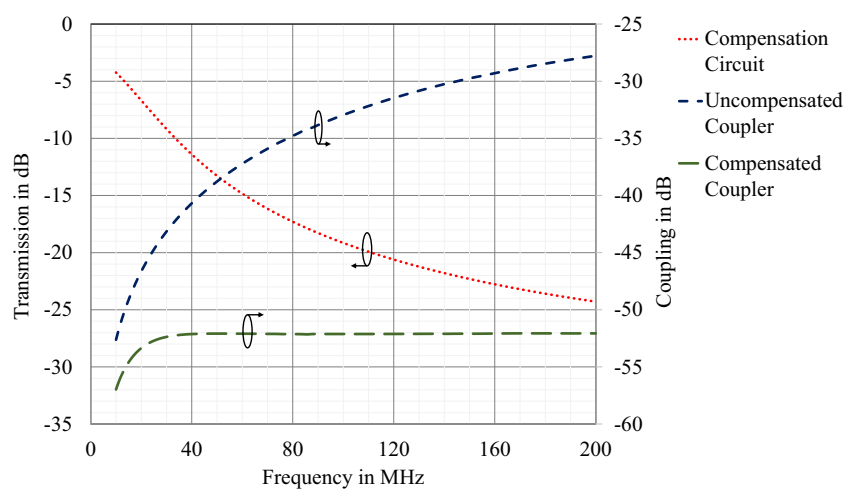

Fig. 7. Coupling of a directional coupler with and without coupling compensation, transmission of the coupling compensation circuit

and the required coupling flatness: The circuit can be seen as the superposition of two attenuators, one for high and one for low frequencies, cf. Fig. 6, so choosing the two attenuations gives the values of the resistors. The value of the capacitor and inductor are then chosen to provide the transition between low and high attenuation in the desired range. Using this technique, the circuit can be used to compensate the directional coupler in Fig. 1a to keep the coupling in the range of $52.11 \mathrm{~dB} \pm 0.05 \mathrm{~dB}$ from $37.4 \mathrm{MHz}$ to $200 \mathrm{MHz}$, cf. Fig. 7 .

\section{Combining directivity and coupling}

Having developed techniques to provide both high directivity and a constant coupling over a frequency range, a circuit to implement both of these behaviours at the same time needs to be found. It has to match the frequency dependent input impedance required for the high directivity to $50 \Omega$ over the bandwidth of interest and introduce a frequency dependent attenuation that counters the increasing coupling inherent in the directional coupler. Obviously, neither putting them in parallel nor in series will work, the networks will have to be integrated into each other. Starting with a coupling compensation network that is designed to match the real part gathered from Fig. 3 to the reference impedance, e.g. $50 \Omega$. To approach the curve of ideal isolation, additional reactive parts need to be added to the circuit which will lead to the existence of a number of possible solutions. One of these solutions can be seen in Fig. 8. Using the techniques proposed in this paper, the directional coupler from Fig. 1a can be 


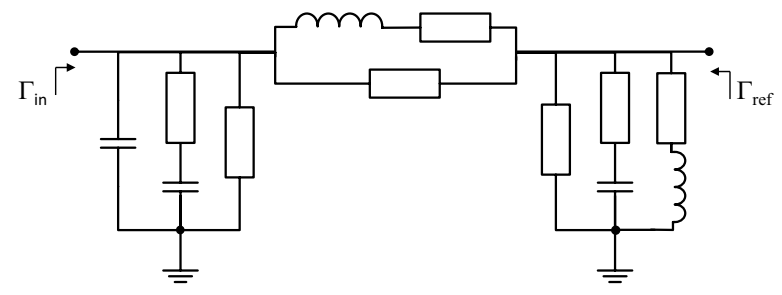

Fig. 8. Example for a full compensation network.

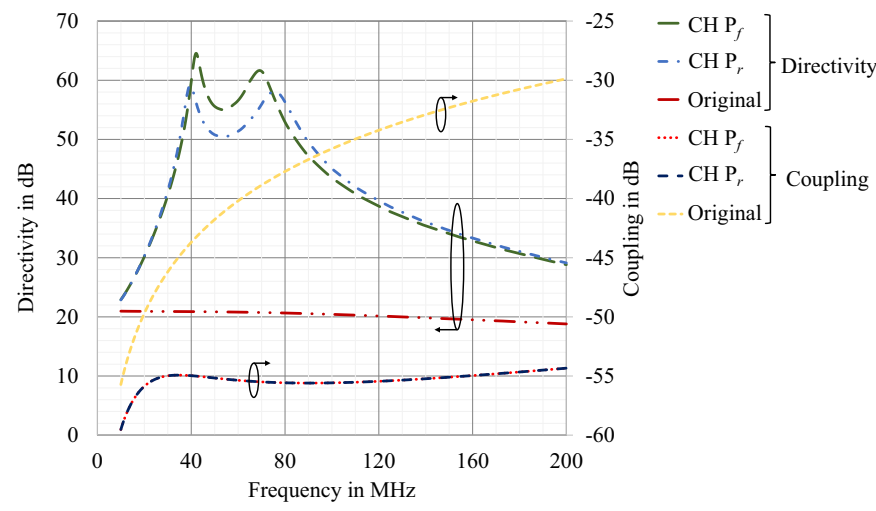

Fig. 9. Coupling and Directivity of the coupler from Fig. 1a with and without compensation.

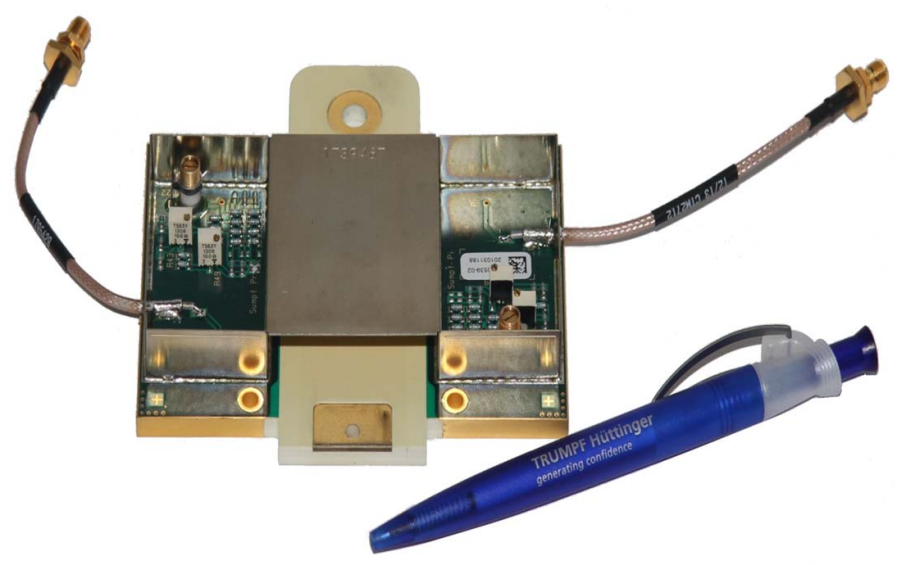

Fig. 10. Example for a TRUMPF Huettinger state of the art compact directional coupler.

equipped with two compensation networks optimised for both directivity and coupling flatness. The network, cf. Fig. 8 is able to keep the coupling within $55.25 \mathrm{~dB} \pm 0.35 \mathrm{~dB}$ from $22 \mathrm{MHz}$ to $164 \mathrm{MHz}$ with a directivity of better than $50 \mathrm{~dB}$ from $35 \mathrm{MHz}$ to $84 \mathrm{MHz}$ and better than $30 \mathrm{~dB}$ over almost a decade from $20 \mathrm{MHz}$ to $187 \mathrm{MHz}$, cf. Fig. 9. Again, the slight difference in s-parameters leads the two directivities to differ.

The proposed strategies have provided the basis for the realisation of compact directional couplers, e.g. the one shown in Fig. 10, to be integrated into future TRUMPF Huettinger RF generators.

\section{CONCLUSION}

With the proposed concept, the directivity and coupling flatness of directional couplers can be improved. The theoretical basis behind the methods used to achieve broadband directivity and coupling is discussed. It has been shown how the impedance for maximum directivity changes with frequency in the scope of directivity enhancements using the cancellation of powers and how a very high directivity can be achieved over a wide bandwidth by designing a network that follows this impedance change. A network has been proposed that allows the compensation of the frequency dependence of a directional couplers coupling. These two concepts were then combined in order to form a network that provides both effects. This concept is particularly of interest in the case of compact microstrip couplers with a single coupling line which can be improved using the proposed techniques to master even the tough challenges in terms of wide band coupling flatness and directivity.

\section{REFERENCES}

[1] S. Uysal and H. Aghvami, "Synthesis, design, and construction of ultra-wide-band nonuniform quadrature directional couplers in inhomogeneous media," Microwave Theory and Techniques, IEEE Transactions on, vol. 37, no. 6, pp. 969-976, 1989.

[2] A. Podell, "A high directivity microstrip coupler technique," in Microwave Symposium, G-MTT 1970 International, 1970, pp. 33-36.

[3] L. Wang, G. Wang, and J. Siden, "Design of high-directivity wideband microstrip directional coupler with fragment-type structure," Microwave Theory and Techniques, IEEE Transactions on, 2015.

[4] J. Muller, M. Pham, and A. Jacob, "Directional coupler compensation with optimally positioned capacitances," Microwave Theory and Techniques, IEEE Transactions on, vol. 59, no. 11, pp. 2824-2832, 2011.

[5] C.-S. Kim, J.-S. Lim, D.-J. Kim, and D. Ahn, "A design of single and multi-section microstrip directional coupler with the high directivity," in Microwave Symposium Digest, 2004 IEEE MTT-S International, vol. 3, 2004, 1895-1898 Vol.3.

[6] S. Lee and Y. Lee, "An inductor-loaded microstrip directional coupler for directivity enhancement," Microwave and Wireless Components Letters, IEEE, vol. 19, no. 6, pp. 362-364, 2009.

[7] V. Tas and A. Atalar, "Using phase relations in microstrip directional couplers to achieve high directivity," Microwave Theory and Techniques, IEEE Transactions on, vol. 61, no. 12, pp. 4063-4071, 2013.

[8] S.-M. Sohn, A. Gopinath, and J. Vaughan, "Tunable and high directivity coupler for MRI applications," in Microwave Symposium (IMS), 2014 IEEE MTT-S International, 2014, pp. 1-3.

[9] E. Mann, C. Gerhardt, C. Thome, C. Wangler, D. Krausse, S. Guenther, and R. Weber. (2012). Measuring power. US Patent 8,203,398, [Online]. Available: http://www.google.co. uk/patents/US8203398. 